\title{
Inelastic effects in Aharonov-Bohm molecular interferometer
}

\author{
Oded $\operatorname{Hod}^{1 \dagger}$, Roi Baer ${ }^{2}$, and Eran Rabani ${ }^{1}$ \\ ${ }^{1}$ School of Chemistry, Tel Aviv University, Tel Aviv 69978, Israel; ${ }^{2}$ Institute of Chemistry, \\ The Hebrew University of Jerusalem, Jerusalem 91904 Israel.
}

(Dated: August 30, 2018)

\begin{abstract}
Inelastic effects arising from electron-phonon coupling in molecular Aharonov-Bohm (AB) interferometers are studied using the nonequilibrium Green's function method. Results for the magnetoconductance are compared for different values of the electron-phonon coupling strength. At low bias voltages, the coupling to the phonons does not change the lifetime and leads mainly to scattering phase shifts of the conducting electrons. Surprisingly, opposite to the behavior of an electrical gate, the magnetoconductance of the molecular AB interferometer becomes more sensitive to the threading magnetic flux as the electron-phonon coupling is increased. PACS numbers: 73.63.-b, 73.63.Fg, $75.75 .+\mathrm{a}$
\end{abstract}

Control of conductance in molecular junctions is of key importance in molecular electronics [1, 2]. The current in these junctions is often controlled by an electrical gate designed to shift conductance peaks into the lowbias regime. Magnetic fields on the other hand, have been rarely used due to the small magnetic flux captured by molecular conductors (an exception is the Kondo effect in single-molecule transistors [3, [4]). This is in contrast to a related field, electronic transport through mesoscopic devices, where considerable activity with magnetic fields has led to the discovery of the quantum hall effect [5] and a rich description of transport in such conductors [6, 7, 8, 9, 10]. The scarcity of experimental activity is due to the belief that significant magnetic response is obtained only when the magnetic flux, $\phi$, is on the order of the quantum flux, $\phi_{0}=h / e$ (where $e$ is the electron charge and $h$ is Planck's constant). Attaining such a flux for molecular and nanoscale devices requires unrealistically huge magnetic fields [1].

Recently, we have described the essential physical requirements necessary for the construction of nanometer scale magnetoresistance devices based on an $\mathrm{AB}$ 12 molecular interferometer [11, 13]. The basic idea was to weakly couple a molecular ring to conducting leads, creating a resonance tunneling junction. The resonant state was tuned by a gate potential to attain maximal conductance in the absence of a magnetic field. The application of a relatively small magnetic field shifts the state out of resonance, and conductance was strongly suppressed within fractions of the quantum flux. The combination of a gate potential and a magnetic field reveals new features and provides additional conductivity control [14, 15].

Our previous study has neglected completely inelastic effects arising from electron-phonon interactions [16, 17, 18, 19, 20, 21, 22]. Can a relatively small magnetic flux change significantly the conduction in molecular rings when the electron-phonon coupling becomes significant? Or, perhaps inelastic effects will broaden the resonant state and conduction will not be suppressed significantly upon the application of the magnetic field. The decay of the amplitude of the $\mathrm{AB}$ oscillations due to electronphonon coupling has been studied for mesoscopic systems [23]. In this letter we address this problem for

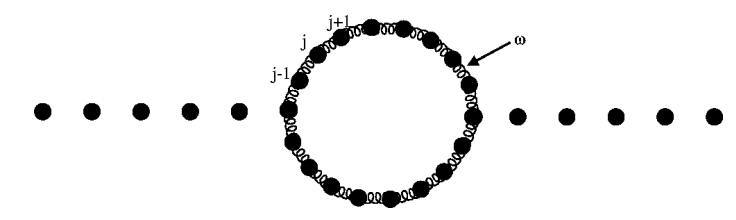

FIG. 1: A sketch of the AB ring. Each site on the ring can be occupied by a single electron. The ring sites are connect by springs with a frequency $\Omega$. An electron on site $j$ is coupled to the local motion of this site, with a coupling strength $M$.

molecular rings, where we focus on the low range of the magnetic flux appropriate for molecular rings. Opposite to the effects of an electrical gate, we find that inelastic effect arising from electron-phonon couplings narrows the magnetoconductance peaks.

We consider a two terminal junction of an AharonovBohm ring with $N$ sites as sketched in Fig. 1 A realization of this model to realistic molecular loops is described elsewhere [1, 13, 14, 15]. We describe the electronic structure of the ring and the leads using a magnetic extended Hückel model [11, 24]. The description of the ring also includes local phonons and electron-phonon interactions are approximated to lowest order. The phonon local frequency $\Omega$ and the coupling $M$ of an electron on site $j$ to the local motion of site $j$ are the only two free parameters of the model. The full Hamiltonian in second quantization is given by:

$$
\begin{aligned}
& H=\sum_{i, j} t_{i, j}(B) c_{i}^{\dagger} c_{j}+\sum_{m, n \in L, R} \epsilon_{m, n}(B) d_{m}^{\dagger} d_{n}+ \\
& \left(\sum_{m, j} V_{m, j}(B) d_{m}^{\dagger} c_{j}+H . c .\right)+\sum_{k=0}^{N-1} \hbar \omega_{k}\left(b_{k}^{\dagger} b_{k}+1 / 2\right) \\
& +\sum_{j, k=0}^{N-1} M_{j}^{k} c_{j}^{\dagger} c_{j}\left(b_{k}^{\dagger}+b_{k}\right) .
\end{aligned}
$$

The first two terms on the right hand side (RHS) of Eq. 11 represent the zero-order electronic Hamiltonian of the ring and leads, respectively. $c_{j}^{\dagger}\left(c_{j}\right)$ is Fermion creation 
(annihilation) operators of an electron on site $j$ on the ring, and $d_{m}^{\dagger}\left(d_{m}\right)$ is Fermion creation (annihilation) operators of an electron on site $m$ on the left (L) or right (R) lead. $t_{i, j}(B)$ and $\epsilon_{i, j}(B)$ are the hopping matrix elements between site $i$ and site $j$ on the ring and lead, respectively. The third term on the RHS of Eq. 1 corresponds to the coupling between the ring and the leads, where $V_{m, j}(B)$ is the hopping element between site $m$ on the lead and site $j$ on the ring. All hopping elements depend on the magnetic field $B$, which is taken to be uniform in the direction perpendicular to the ring plane. Both linear and quadratic terms in the magnetic field are included in the calculation [11]. The last two terms in Eq. 1 represents the Hamiltonian of the phonons and the electron-phonon interactions. $b_{k}^{\dagger}\left(b_{k}\right)$ is a boson creation (annihilation) operator of phonon mode $k$ with a corresponding frequency $\omega_{k}$. This set of phonon modes was obtained by a unitary transformation from local to normal coordinates of a one dimensional chain of coupled harmonic oscillators, characterized by a single frequency $\Omega$, as illustrate in Fig. 1] These frequencies constitute a band of width proportional to the coupling between the oscillators. The electron-phonon coupling is approximated to lowest order. Each site on the ring is coupled to all phonon modes with a coupling strength $M_{j}^{k}=M \sqrt{\frac{\Omega}{\omega_{k}}} U_{j k}$, where $U_{j k}$ are the matrix elements of transformation matrix $\mathbf{U}$ from local to normal modes.

The calculation of the conductance is described within the framework of the nonequilibrium Green's function (NEGF) method 25]. The total current $I=I_{e l}+I_{\text {inel }}$ is recast as a sum of elastic $\left(I_{e l}\right)$ and inelastic $\left(I_{\text {inel }}\right)$ contributions given by $21,22,26]$

$$
\begin{array}{r}
I_{e l}=\frac{2 e}{\hbar} \int \frac{d \epsilon}{2 \pi}\left[f\left(\epsilon, \mu_{R}\right)-f\left(\epsilon, \mu_{L}\right)\right] \\
\operatorname{Tr}\left[\boldsymbol{\Gamma}_{L}(\epsilon) \mathbf{G}^{r}(\epsilon) \boldsymbol{\Gamma}_{R}(\epsilon) \mathbf{G}^{a}(\epsilon)\right]
\end{array}
$$

and

$$
\begin{gathered}
I_{\text {inel }}=\frac{2 e}{\hbar} \int \frac{d \epsilon}{2 \pi} \operatorname{Tr}\left[\boldsymbol{\Sigma}_{L}^{<}(\epsilon) \mathbf{G}^{r}(\epsilon) \boldsymbol{\Sigma}_{p h}^{>}(\epsilon) \mathbf{G}^{a}(\epsilon)\right. \\
\left.-\boldsymbol{\Sigma}_{L}^{>}(\epsilon) \mathbf{G}^{r}(\epsilon) \boldsymbol{\Sigma}_{p h}^{<}(\epsilon) \mathbf{G}^{a}(\epsilon)\right]
\end{gathered}
$$

respectively. The retarded (advanced) GFs satisfy the Dyson equation

$$
\mathbf{G}^{r, a}(\epsilon)=\left\{\left[\mathbf{g}^{r, a}(\epsilon)\right]^{-1}-\boldsymbol{\Sigma}_{L}^{r, a}(\epsilon)-\boldsymbol{\Sigma}_{R}^{r, a}(\epsilon)-\boldsymbol{\Sigma}_{p h}^{r, a}(\epsilon)\right\}^{-1},
$$

where $\mathbf{g}^{r, a}(\epsilon)$ is the uncoupled retarded (advanced) electronic GF of the ring. The greater (lesser) GFs satisfy the Keldysh equation at steady state (for an initial noninteracting state)

$$
\mathbf{G}^{\lessgtr}(\epsilon)=\mathbf{G}^{r}(\epsilon)\left[\boldsymbol{\Sigma}_{L}^{\lessgtr}(\epsilon)+\boldsymbol{\Sigma}_{R}^{\lessgtr}(\epsilon)+\boldsymbol{\Sigma}_{p h}^{\lessgtr}(\epsilon)\right] \mathbf{G}^{a}(\epsilon) .
$$

In the above equations, $\boldsymbol{\Sigma}_{L}^{r, a, \lessgtr}(\epsilon), \quad \boldsymbol{\Sigma}_{R}^{r, a, \lessgtr}(\epsilon), \quad$ and $\boldsymbol{\Sigma}_{p h}^{r, a, \lessgtr}(\epsilon)$ are the retarded $(r)$, advanced $(a)$, lesser $(<)$

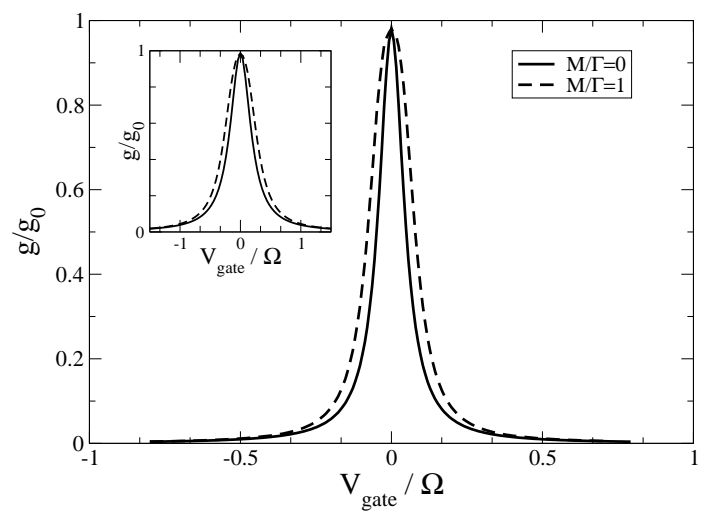

FIG. 2: Conduction as a function of the gate voltage at zero magnetic flux with (dashed line) and without (solid line) electron-phonon coupling. Inset: A similar plot for the single resonant level model described in Ref. 22 .

and greater $(>)$ self-energies arising from the coupling to the left lead, right lead, and the phonons, respectively, and $\boldsymbol{\Gamma}_{\mathrm{L}, \mathrm{R}}(\epsilon)=i\left[\boldsymbol{\Sigma}_{\mathrm{L}, \mathrm{R}}^{r}(\epsilon)-\boldsymbol{\Sigma}_{\mathrm{L}, \mathrm{R}}^{a}(\epsilon)\right]$, where

$$
\boldsymbol{\Sigma}_{\mathrm{L}, \mathrm{R}}^{r, a}(\epsilon)=\left(\epsilon \mathbf{S}^{*}-\mathbf{V}^{*}(B)\right) \mathbf{g}_{\mathrm{L}, \mathrm{R}}^{r, a}(\epsilon)(\epsilon \mathbf{S}-\mathbf{V}(B)) .
$$

In the above, $\mathbf{V}(B)$ is the lead-ring hopping matrix with elements $V_{m j}(B), \mathbf{S}$ is the overlap matrix between the states on the leads and on the ring, and $\mathbf{g}_{\mathrm{L}, \mathrm{R}}^{r, a}(\epsilon)$ is the retarded (advanced) uncoupled GF of the left or right lead. The corresponding leaser (greater) self-energies are given by

$$
\boldsymbol{\Sigma}_{\mathrm{L}, \mathrm{R}}^{\lessgtr}(\epsilon)=\left(\delta_{\lessgtr}-f\left(\epsilon, \mu_{\mathrm{L}, \mathrm{R}}\right)\right)\left[\boldsymbol{\Sigma}_{\mathrm{L}, \mathrm{R}}^{r}(\epsilon)-\boldsymbol{\Sigma}_{\mathrm{L}, \mathrm{R}}^{a}(\epsilon)\right],
$$

where $\delta_{\lessgtr}$ equals 0 for $<$ and 1 otherwise, and $f(\epsilon, \mu)=$ $\frac{1}{1+e^{\beta(\epsilon-\mu)}}$. The self-energy arising from the interactions with the phonons is calculated using the first Born approximation (FBA) and is given by [21, 22, 26]:

$$
\begin{aligned}
\boldsymbol{\Sigma}_{p h}^{r}(\epsilon)= & i \sum_{k} \int \frac{d \omega}{2 \pi} \mathbf{M}^{k}\left\{D_{k}^{<}(\omega) \mathbf{g}^{r}(\epsilon-\omega)+\right. \\
& \left.D_{k}^{r}(\omega) \mathbf{g}^{<}(\epsilon-\omega)+D_{k}^{r}(\omega) \mathbf{g}^{r}(\epsilon-\omega)\right\} \mathbf{M}^{k},
\end{aligned}
$$

where the Hartree term has been omitted 22]. The lesser and greater self energies arising from the coupling to the phonons are given by:

$$
\boldsymbol{\Sigma}_{p h}^{\lessgtr}(\epsilon)=i \sum_{k} \int \frac{d \omega}{2 \pi} \mathbf{M}^{k} D_{k}^{\lessgtr}(\omega) \mathbf{g}^{\lessgtr}(\epsilon-\omega) \mathbf{M}^{k} .
$$

In the above equations, $D_{k}^{r, a}$ and $D_{k}^{\lessgtr}$ are the uncoupled equilibrium retarded (advanced) and lesser (greater) GFs of phonon mode $k$, respectively, $\mathbf{g}^{\lessgtr}(\epsilon)$ is the lesser (greater) uncoupled electronic GF of the ring, and $\mathbf{M}^{k}$ is the electron-phonon coupling matrix of mode $k$ (diagonal in the $c_{j}$ basis).

We now turn to discuss the results of a specific realization of the above model. We consider a ring composed of 
$N=40$ sites. The sites are identical and contribute a single electron which is described by a single Slater $s$-like orbital. The coupling between the ring and the leads is limited to the contact region. For simplicity, the electronic self-energies arising from this coupling are approximated within the wide band limit. Specifically, we neglect the real-part of the electronic self-energy and approximate $\boldsymbol{\Gamma}_{L, R}(\epsilon)$ with matrices that are independent of energy, where the only non-vanishing elements are the diagonal elements $\left(\Gamma_{L, R}\right)$ corresponding to the two sites coupled to the left or right lead. The local phonon frequency $\Omega=0.0125 \mathrm{eV}$ is characteristic of a low frequency optical phonon in molecular devices. Since our model does not include a secondary phonon bath required to relax the energy from the optical phonons, we include a phonon energy level broadening $\eta=0.016 \Omega$ which is included in the uncoupled GFs of the phonon. The coupling to each of the leads is taken to be $\Gamma_{L}=\Gamma_{R}=4 \Omega$ such that the magnetoconductance switching in the absence of electron-phonon coupling is obtained at $\sim 5$ Tesla.

Before we address the effects of electron-phonon coupling on the magnetoconductance properties of the system described above we will analyze the role of a gate potential on the conductance. In Fig. 2 we plot the zero-bias conduction as a function of a gate voltage with $\left(M / \Gamma=1\right.$, where $\left.\Gamma=\Gamma_{L}+\Gamma_{R}\right)$ and without $(M / \Gamma=0)$ electron-phonon coupling. The gate voltage was modeled by an additional potential $e V_{g} \mathbf{S}$, where $\mathbf{S}$ is the overlap matrix, that was added to the ring hopping matrix element $t_{i j}$. For comparison (inset of Fig. 2) we also include the results of a single resonant level coupled to a single phonon with identical model parameters used by Mitra et al. [22].

The two most significant observations are the expected broadening of the conduction when the electron-phonon coupling is turned on and the value of the zero-bias conduction $\left(g / g_{0}=1\right.$, where $g_{0}=2 e / h$ is the quantum conductance) in the presence of electron-phonon coupling. To better understand these results we rewrite the current for the case that $\boldsymbol{\Gamma}_{L}(\epsilon)=\boldsymbol{\Gamma}_{R}(\epsilon) \equiv \boldsymbol{\Gamma}(\epsilon) / 2$ in the following way [27]: $I=\frac{2 e}{h} \int d \epsilon\left(f\left(\epsilon-\mu_{L}\right)-f\left(\epsilon-\mu_{R}\right)\right) \mathcal{T}(\epsilon)$ where $\mathcal{T}(\epsilon)=\frac{i}{4} \operatorname{Tr} \boldsymbol{\Gamma}(\epsilon)\left(\mathbf{G}^{r}(\epsilon)-\mathbf{G}^{a}(\epsilon)\right)$. Note that $\mathcal{T}(\epsilon)$ is the transmission coefficient only when $M=0$. In the wide band limit, for the single resonant level model, $\mathcal{T}(\epsilon)$ can be reduced to $\frac{\Gamma}{4} \frac{\Gamma-2 \Sigma_{p h, i m}^{r}(\epsilon)}{\left(\epsilon-\epsilon_{0}-\Sigma_{p h, r e}^{r}(\epsilon)\right)^{2}+\left(\Gamma / 2-\Sigma_{p h, i m}^{r}(\epsilon)\right)^{2}}$. As a result of the fact that $\Sigma_{p h, i m}^{p h, r e}(0)=0$ at zero bias, the only inelastic contribution to the conduction comes from the real part of the phonon self-energy [22]. From this, it follows that even in the presence of electron-phonon coupling, the maximal conduction is $g_{\max } / g_{0}=1$, as clearly can be seen in Fig. 2 for both cases. It also immediately implies that the main contribution to the broadening of the resonant conduction peak comes from the real-part of the phonon self-energy, i.e., from processes that lead to scattering phase shifts, but do not change the lifetime of the state.

So far we have discussed the effect of electron-phonon

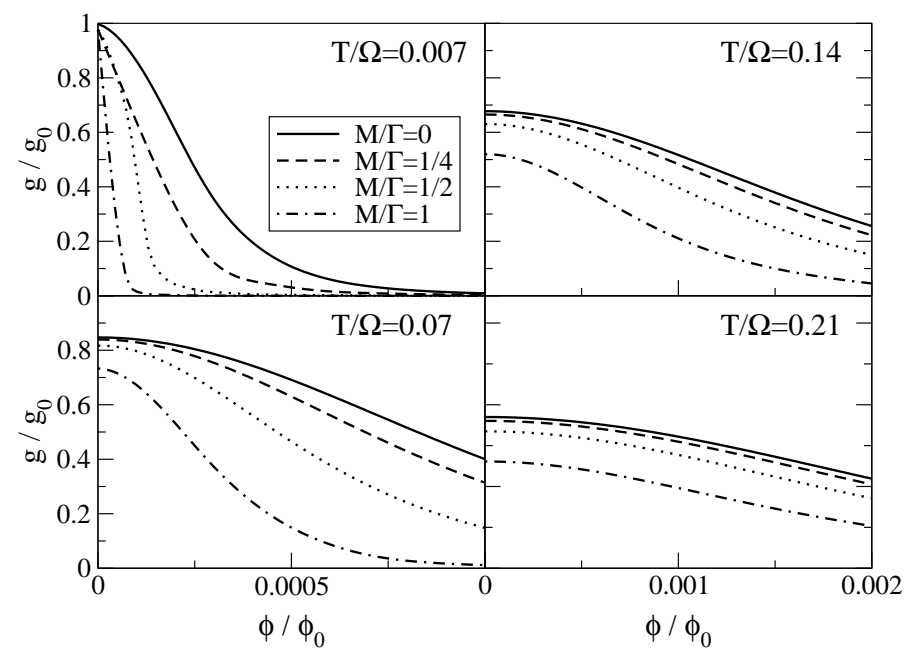

FIG. 3: Conductance as a function of magnetic flux for several values of the electron-phonon coupling strength $M$ and for different temperatures.

coupling on the zero-bias conduction as a function of a gate voltage. We now turn to discuss the major result of the present study. In Fig. 3 we plot the magnetoconductance of the AB-ring for several values of the electron-phonon couplings $(M)$ and for different temperatures $(T)$. We focus on the low value of the magnetic flux $\phi=A B$, where $A$ is the area of the ring and $B$ is taken perpendicular to the ring plane.

The case $M=0$ for different systems was discussed in detail in our previous studies, where the main goal was to establish the conditions required to achieve negative magnetoconductance and magnetic switching at low magnetic fields, despite the relatively large magnetic fields required to complete a full $\mathrm{AB}$ period [11, 13, 15]. The essential procedure described in 11] was to weakly couple the ABring to the conducting leads and at the same time to apply a gate potential to shift the position of the resonance state such that conduction is maximized at $\phi / \phi_{0}=0$. A manifestation of these ideas is depicted in Fig. 3 for the case that $M=0$ (solid curves), where the conduction is reduced from its maximal value to a small value at a relatively low magnetic flux. As expected, we find that as the temperature is increased the maximal value $g / g_{0}$ is decreased and the width of the magnetoconductance peaks is increased linearly with $T$ for $M / \Gamma=0$ (with deviations from linearity as $M / \Gamma$ is increased). This increase in the width with temperature is a result of resonant tunneling and the broadening of the Fermi distributions as $T$ is varied.

Turning to discuss the case of $M \neq 0$, one of the major questions is related to the effects of electron-phonon coupling on the switching capability of small AB-rings. Based on the discussion of the results shown in Fig. 22 one might expect that an increase in $M$ will lead to a broadening of the magnetoconductance peaks, thereby increas- 


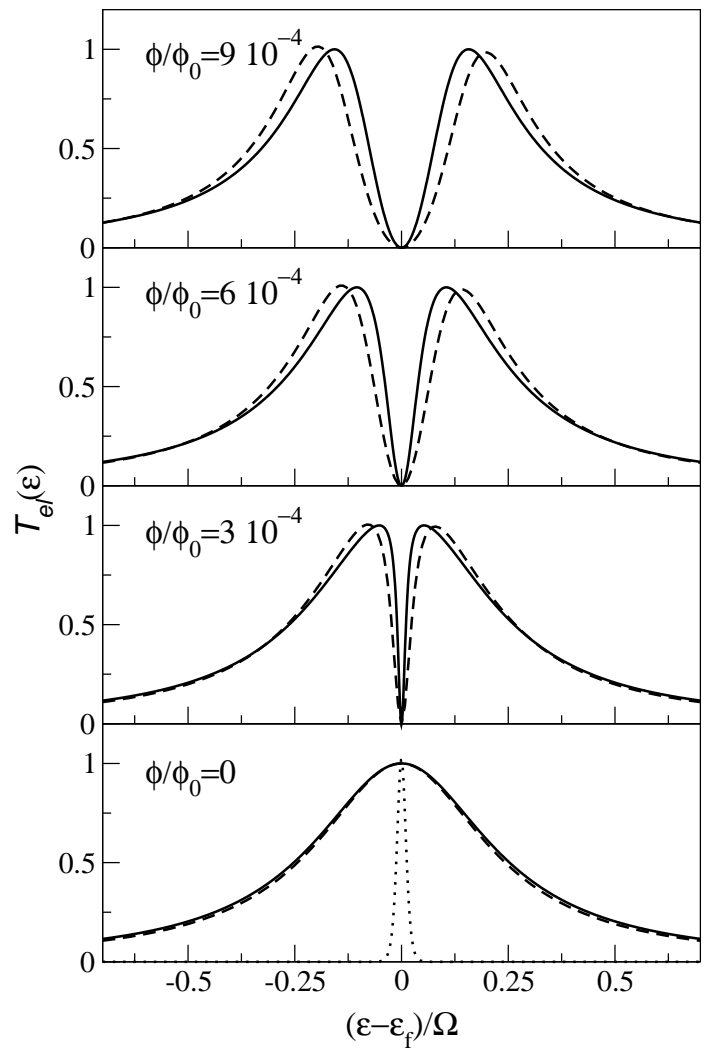

FIG. 4: Plots of $\mathcal{T}(\epsilon)$ as a function of energy for $M / \Gamma=0$ (solid curves) and $M / \Gamma=\frac{1}{4}$ (dashed curves) for different values of the magnetic flux. The dotted curve at the lower panel shows $\frac{\partial}{\partial \mu} \Delta f(\epsilon-\mu)$ at $T / \Omega=0.007$, where $\Delta f(\epsilon-\mu)$ is the difference in the Fermi distribution of the left and right lead.

ing the value of the magnetic field required to switch a nanometer AB-ring, and perhaps leads to unphysical values of $B$ required to reduced the conduction significantly. As can be seen in Fig. [3] the numerical solution of the NEGF for $M \neq 0$ leads to a reduction of the width of the magnetoconductance peaks, and the switching of the AB-ring is achieved at lower values of the magnetic flux compared to the case where $M=0$.

This surprising observation can be explained in simple terms. As discussed above, even in the presence of electron-phonon coupling, the maximal conduction at zero bias and zero temperature is $g_{\max } / g_{0}=1$, as clearly is the case for the results shown in the upper left panel of Fig. 3 for $\phi / \phi_{0}=0$. For the symmetric ring of $N=4 n$ the resonance condition at $\phi / \phi_{0}=0$ is equivalent to the condition that electrons entering the ring from left interfere constructively when they exit the ring to the right [11, 13]. This picture also holds when $M \neq 0$, and the conduction takes a maximal value at $\phi / \phi_{0}=0$. The application of a magnetic field leads to destructive in- terference and increases the back scattering of electrons. This loss of phase is even more pronounced when inelastic effects arising from electron-phonon coupling are included. In the magnetoconductance this is translated to a more rapid loss of conduction as a function of the magnetic field when $M$ is increased.

Mathematically, the rapid decay of the conduction with the magnetic field as the electron-phonon coupling is increased can be explained by analyzing the dependence of $\mathcal{T}(\epsilon)$. In Fig. 4 we plot $\mathcal{T}_{\text {el }}(\epsilon)=$ $\operatorname{Tr}\left[\boldsymbol{\Gamma}_{L}(\epsilon) \mathbf{G}^{r}(\epsilon) \boldsymbol{\Gamma}_{R}(\epsilon) \mathbf{G}^{a}(\epsilon)\right]$, which is elastic (and dominant) contribution to $\mathcal{T}(\epsilon)$, as a function of energy for several values of $\phi / \phi_{0}$ for $M / \Gamma=0$ or $M / \Gamma=\frac{1}{4}$. In the lower panel we also plot the corresponding Fermi distribution window. At $\phi / \phi_{0}=0, \mathcal{T}_{e l}(\epsilon) \approx 1$ near the Fermi energy $\left(\epsilon_{f}\right)$, independent of $M$, and the conduction is $g / g_{0} \approx 1$. The application of a small magnetic field results in a split of $\mathcal{T}_{e l}(\epsilon)$, where each peak corresponds to a different circular state [28]. The separation between the two peaks in the elastic limit $\Delta=\left(\epsilon_{2}-\epsilon_{1}\right) \propto \phi / \phi_{0}$ is proportional to the magnetic flux, where $\epsilon_{1,2}$ are the corresponding energies of the two circular states. When inelastic terms are included, due to the fact that the imaginary part of $\boldsymbol{\Sigma}_{p h}^{r}(\epsilon)$ is negligibly small, the renormalized positions of the two peaks can be approximated by $\epsilon_{1,2}^{*}=\epsilon_{1,2}+\Sigma_{p h, r e}^{r}\left(\epsilon_{1,2}\right)=\epsilon_{1,2} \pm \Sigma_{p h, r e}^{r}\left(\epsilon_{2}\right)$, which implies that the renormalized separation between the two peaks can be approximated by $\Delta^{*}=\left(\epsilon_{2}^{*}-\epsilon_{1}^{*}\right)=\Delta+2 \Sigma_{p h, r e}^{r}\left(\epsilon_{2}\right)$. Therefore, as $M$ is increased $\Delta^{*}$ is also increased, consis-

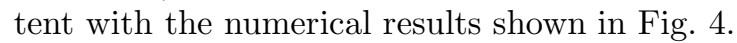

Similarly to the electrical gate, the magnetic field provides means to externally control the conductance of a ring-shaped molecular junction. However, there are striking differences in the properties of these two gauges. This was illustrated previously in a multi-terminal device, where the polarity of the magnetic field, which couples to the electronic angular momentum, played a key role. In the present study we showed that there is also a fundamentally difference with respect to inelastic effects. While the conductance as a function of the gate voltage broadens due to coupling to phonons it actually narrows considerably in response to a magnetic field. This unexpected result was rationalized in terms of a rapid loss of the phase of electrons at the exit channel arising from the coupling to the phonons. Mathematically, this effect was traced to the form of the real part of the phonon self-energy that gives rise to scattering phase shifts, but does not change the lifetime of the resonant level through which conduction takes place.

We thank Abe Nitzan for many fruitful discussions. O.H. would like to thank the generous financial support of the Rothschild and Fulbright foundations. This work was supported by the Israel Science Foundation (grants to E.R. and R.B.). 'Present address: Department of Chemistry, Rice University, Houston, TX 77251-1892. 
[1] C. Joachim, J. k. Gimzewski, and A. Aviram, Nature 408, 541 (2000).

[2] A. Nitzan and M. A. Ratner, Science 300, 1384 (2003).

[3] J. Park et al., Nature 417, 722 (2002).

[4] W. J. Liang et al., Nature 417, 725 (2002).

[5] K. von Klitzing, G. Dorda, and M. Pepper, Phys. Rev. Lett. 45, 494 (1980).

[6] R. A. Webb, S. Washburn, C. P. Umbach, and R. B. Laibowitz, Phys. Rev. Lett. 54, 2696 (1985).

[7] G. Timp et al., Phys. Rev. Lett. 58, 2814 (1987).

[8] A. Yacoby, M. Heiblum, D. Mahalu, and H. Shtrikman, Phys. Rev. Lett. 74, 4047 (1995).

[9] A. van Oudenaarden, M. H. Devoret, Y. V. Nazarov, and J. E. Mooij, Nature 391, 768 (1998).

[10] A. Fuhrer et al., Nature 413, 822 (2001).

[11] O. Hod, R. Baer, and E. Rabani, J. Phys. Chem. B 108, 14807 (2004).

[12] Y. Aharonov and D. Bohm, Phys. Rev. 115, 485 (1959).

[13] O. Hod, E. Rabani, and R. Baer, J. Chem. Phys. 123, 051103 (2005).

[14] O. Hod, R. Baer, and E. Rabani, J. Am. Chem. Soc. 127, 1648 (2005).

[15] O. Hod, E. Rabani, and R. Baer, Acc. Chem. Res. 39, 109 (2006).
[16] H. Ness and J. Fisher, Phys. Rev. Lett. 83, 452 (1999).

[17] D. Segal and A. Nitzan, Chem. Phys. 281, 235 (2002).

[18] M. J. Montgomery, T. N. Todorov, and A. P. Sutton, J. Phys.: Condens. Matter 14, 5377 (2002).

[19] E. G. Petrov, V. May, and P. Hanggi, Chem. Phys. 296, 251 (2003).

[20] T. Mii, S. G. Tikhodeev, and H. Ueba, Phys. Rev. B 68, 205406 (2003).

[21] M. Galperin, M. A. Ratner, and A. Nitzan, J. Chem. Phys. 121, 11965 (2004).

[22] A. Mitra, I. Aleiner, and A. J. Millis, Phys. Rev. B 69, 245302 (2004).

[23] F. Guinea, Phys. Rev. B 65, 205317 (2002).

[24] O. Hod, Ph.D. thesis, Tel Aviv University, 2005.

[25] S. Datta, Electronic Transport in Mesoscopic Systems (Cambridge University Press, Cambridge, 1995).

[26] M. Paulsson, T. Frederiksen, and M. Brandybge, Phys. Rev. B 72, 201101(R) (2005).

[27] A. P. Jauho, N. S. Wingreen, and Y. Meir, Phys. Rev. B 50, 5528 (1994).

[28] A. Silva, Y. Oreg, and Y. Gefen, Phys. Rev. B 66, 195316 (2002). 